\title{
Investigation of moisture stability and PL characteristics of terpineol-passivated organic-inorganic hybrid perovskite
}

\author{
Xin Guo ${ }^{1,2} \cdot$ Christopher McCleese $^{1} \cdot$ Weiyin Gao ${ }^{1,3} \cdot$ Minqiang Wang $^{3} \cdot$ \\ Lixia Sang ${ }^{4}$ Clemens Burda ${ }^{1,2}$
}

Received: 22 April 2016/Accepted: 30 August 2016/Published online: 22 September 2016

(c) The Author(s) 2016. This article is published with open access at Springerlink.com

\begin{abstract}
This work presents a novel method for preparing perovskite films using a simple processing technique. Perovskite paste was prepared by dispersing an equimolar mix of $\mathrm{PbI}_{2}$ and methyl ammonium iodide powders into terpineol with stirring. From these precursors, perovskite films were fabricated using doctor blading and drying for $24 \mathrm{~h}$ at room temperature. The prepared films were then placed into relative humidity (RH) levels of 30,50 , and $70 \%$ to test the moisture stability. The crystal structure, phases, and morphology were investigated with XRD and SEM/EDX. These samples exhibited good stability against long time exposure to moisture for 70 days. The XRD results showed that samples stored at $\mathrm{RH} 70 \%$ contained only a small amount of hydrate compound after 70 days storage, while in the sample stored at RH $50 \%$, the formation of $\mathrm{PbI}_{2}$ was observed. The sample at $\mathrm{RH} 30 \%$ manifested almost no change when stored for the same
\end{abstract}

Electronic supplementary material The online version of this article (doi:10.1007/s40243-016-0081-1) contains supplementary material, which is available to authorized users.

Clemens Burda

burda@case.edu

1 Department of Chemistry, Case Western Reserve University, 10900 Euclid Avenue, Cleveland, OH 44106, USA

2 Department of Materials Science and Engineering, Case Western Reserve University, 10900 Euclid Avenue, Cleveland, OH 44106, USA

3 Electronic Materials Research Laboratory, Key Laboratory of Ministry of Education, International Centers for Dielectric Research, Xi' an Jiaotong University, Xi' an 710049, China

4 Key Laboratory of Enhanced Heat Transfer and Energy Conservation, Ministry of Education, Key Laboratory of Heat Transfer and Energy Conversion, Beijing Municipality, College of Environmental and Energy Engineering, Beijing University of Technology, Beijing 100124, China storage period. We attribute the enhanced moisture stability, compared with the spin-coated samples, to a passivated surface of the perovskite film by terpineol which exhibits a hydrophobic moiety. Time-resolved photoluminescence measurements show that the passivation of surface defect states by the formation of either $\mathrm{PbI}_{2}$ or hydrated compound leads to prolonged charge carrier recombination times.

\section{Graphical Abstract}

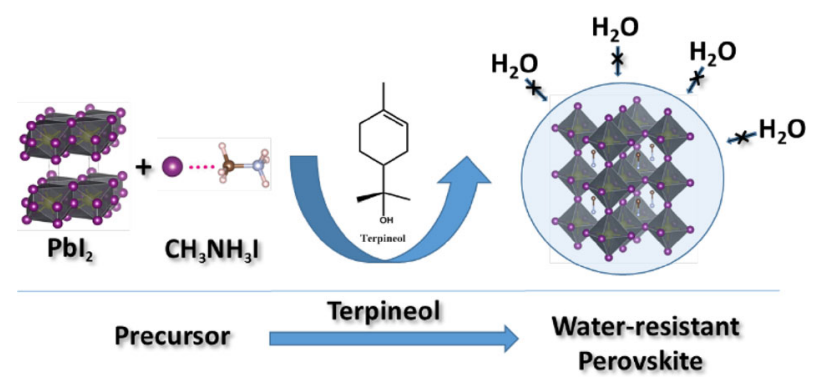

Keywords Perovskite paste - Terpineol passivation . Moisture stability · Humidity degradation · PL decay lifetimes

\section{Introduction}

Over the last few years, solar cells based on organicinorganic hybrid semiconductor materials with perovskite structure as light absorbers have been quickly progressing due to their excellent photovoltaic performance [1-3]. Perovskites were first utilized in dye sensitized solar cells with the perovskite being a replacement sensitizer for the standard molecular dyes. Kojima et al. reported the power

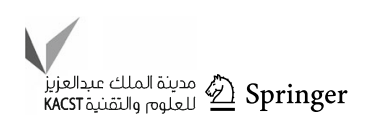


Table 1 PL decay lifetimes of fresh and aged perovskite films

\begin{tabular}{lclr}
\hline Sample & Day & $\tau_{1} / \mathrm{ns}$ & $\tau_{2} / \mathrm{ns}$ \\
\hline Fresh & 0 & - & $16.9 \pm 0.1$ \\
$30 \% \mathrm{RH}$ & 3 & $3.0 \pm 0.1(0.82)$ & $13.0 \pm 1.0(0.18)$ \\
& 10 & $4.2 \pm 0.3(0.78)$ & $14.0 \pm 1.0(0.22)$ \\
$50 \% \mathrm{RH}$ & 30 & $4.8 \pm 0.3(0.75)$ & $15.0 \pm 1.0(0.25)$ \\
& 3 & $4.2 \pm 0.2(0.82)$ & $15.0 \pm 1.0(0.18)$ \\
$70 \% \mathrm{RH}$ & 10 & $3.7 \pm 0.2(0.87)$ & $15.0 \pm 1.0(0.13)$ \\
& 30 & $7.0 \pm 0.2(0.84)$ & $36.0 \pm 2.0(0.16)$ \\
& 3 & $3.8 \pm 0.1(0.89)$ & $17.0 \pm 2.0(0.11)$ \\
& 10 & $3.7 \pm 0.1(0.87)$ & $18.0 \pm 2.0(0.13)$ \\
& 30 & $5.1 \pm 0.1(0.81)$ & $22.0 \pm 1.0(0.19)$ \\
\hline
\end{tabular}

conversion efficiency (PCE) of a $\mathrm{CH}_{3} \mathrm{NH}_{3} \mathrm{PbI}_{3}$ perovskite solar cell (PSC) of $3.81 \%$. However, incorporating perovskite into a solid-state architecture has greatly improved efficiencies, and to date, the highest certified PCE is $20.1 \%$ [4]. This class of materials is advantageous for solar-cell applications, particularly with respect to their low processing cost, simple and various synthetic methods, and high power conversion efficiency [4-7]. These organic-inorganic hybrid semiconductors can be fabricated from relatively inexpensive materials by a simple solution process without annealing at high temperature, and their cost could be considerably reduced through mass production [8-11]. Moreover, solar cells based on metal halide perovskites have multiple advantages: excellent optical properties with tunable light absorption by managing the chemical composition, low exciton-binding energy with the excited state composed primarily of free carriers, fast photogeneration, ambipolar charge transport, excellent charge carrier mobility, and long electron-hole diffusion lengths [12-17].

Although efficiency progress and photovoltaic performance of perovskite-based solar devices have been remarkable, the lead-halide perovskites have been suffering due to their instability when exposed to atmospheric moisture. The intercalated polar methyl ammonium ion, $\mathrm{CH}_{3} \mathrm{NH}_{3}{ }^{+}$, in the perovskite has a tendency to diffuse inside the perovskite framework with the aid of a polar solvent like water. The $\mathrm{CH}_{3} \mathrm{NH}_{3} \mathrm{PbI}_{3}$ perovskite films will easily degrade into other chemical species when it is exposed to water (humidity), resulting in an intrinsic problem, regarding long-term stability [18-22]. Only limited research has been conducted on enhancing the stability and requires immediate attention if perovskite-based photovoltaic devices are to be used commercially.

It has been shown that a passivation layer is a good strategy to provide an enhanced stability for perovskites.
Recent published work [23] has also confirmed that $\mathrm{PbI}_{2}$ with a bulk band gap of $2.3 \mathrm{eV}$ can form a passivation layer in the unfilled grain boundaries of perovskite films. The slower injection rate and longer recombination times of photoinduced charge carriers demonstrate the effect of passivation. The post-modification of perovskite by aluminum oxide reported by Wang et al. [21] could both protect the perovskite sensitizer from degradation and effectively suppress electron recombination. Snaith et al. have also reported significantly reduced electron-hole recombination and longer carrier lifetime $\sim 2 \mu$ s within the $\mathrm{CH}_{3} \mathrm{NH}_{3} \mathrm{PbI}_{3-\mathrm{x}} \mathrm{Cl}_{\mathrm{x}}$ perovskite through the treatment of the crystal surfaces with the Lewis bases thiophene and pyridine [24]. It is found that there is an excess of charge building up by under-coordinated $\mathrm{Pb}$ atoms at crystal surfaces and grain boundaries. The electronic passivation by forming coordinate or dative bonds between unsaturated $\mathrm{Pb}$ ions and Lewis bases could effectively mitigate the charge build-up at defect sites and enhance the stability of the perovskite crystals along with power conversion efficiencies for the Lewis Base treated solar cells. A recent study by Karunadasa et al. demonstrated the enhanced moisture stability of a layered 2D hybrid perovskite [25], in which phenylethylamine (PEA) not only acts as a molecule for tuning the spacing between the perovskite layers toward higher bandgap, but also provides the perovskite absorber with a hydrophobic surface, which leads to increased durability. Yong et al. introduced a simple and effective passivation method to overcome water vulnerability for the perovskite solar cell [26]. By coating a hydrophobic polytetrafluoroethylene (PTFE) layer onto the top surface of the cell, water diffusion into the perovskite cell was effectively blocked and the solar cell exhibited a significantly enhanced stability under the ambient atmosphere conditions. 
In this work, we introduce a new hydrophobic molecule in a new passivation process. By blading the perovskite paste, the modified perovskite film presented significantly enhanced moisture stability. Water molecule penetration into the perovskite crystal lattice was prevented chemically by the hydrophobic repulsion at the molecular level and physically by the layer encapsulation on a macroscopic scale. Through the hydrophobic passivation, a good carrier lifetime measured by time-resolved photoluminescence (TRPL) decay indicates less trap states formation and good electron transport with terpineol modification. We also present different degradation processes of samples at three different humidity levels, and traced it by the XRD analysis. SEM/EDX analysis was used to confirm morphology and compositional change during phase degradation.

\section{Experimental methods}

\section{Materials}

Methyl ammonium iodide (MAI) powders were synthesized by reacting a concentrated aqueous solution of hydroiodic acid (57 wt \%, Aldrich) with methylamine (33 $\mathrm{wt} \%$ in methanol) in an ice bath for $2 \mathrm{~h}$ with stirring. The mixture was dried at $65{ }^{\circ} \mathrm{C}$ for $30 \mathrm{~min}$ using a rotary evaporator. The precipitant obtained was washed three times with diethyl ether and dried in a vacuum oven at $60{ }^{\circ} \mathrm{C}$ for $24 \mathrm{~h}$. Commercial powders of $\mathrm{PbI}_{2}$ and $\alpha$-Terpineol were used as received from Sigma-Aldrich.

\section{Synthesis of $\mathrm{MAPbI}_{3}$}

$\mathrm{MAPbI}_{3}$ perovskite film samples were prepared by doctor blading the perovskite paste. The mixture of $\mathrm{PbI}_{2}$ and MAI with a molar ratio of $1: 1$ was first dispersed in $\alpha$-Terpineol to form a 2.5:1 paste. The perovskite paste was finally produced by magnetic stirring for 3 days. The bladed $\mathrm{MAPbI}_{3}$ perovskite films were dried at room temperature for $24 \mathrm{~h}$.

\section{Moisture stability of $\mathrm{MAPbI}_{3}$ film}

The stability of the bladed perovskite thin films on the glass substrates was tested by placing samples at three different RH levels: 30,50 , and $70 \%$ for 70 days. The degraded conditions were tracked in $3,10,30$, and 70 days using $\mathrm{X}$-ray diffraction and scanning electron microscopy (SEM). The carrier lifetime of the 3, 10, and 30 days samples at each RH level was measured by time-resolved photoluminescence spectroscopy.

\section{Materials characterization}

X-Ray diffraction (XRD) patterns were obtained with a Rigaku MiniFlex instrument $\mathrm{Cu} \mathrm{K} \alpha$ beam $(\lambda=1.54 \AA)$. SEM images and energy dispersive X-ray spectroscopy (EDX) spectra were acquired by Phenom ProX. Thermo Nicolet NEXUS 870 FT-IR spectrometer was used to collect the FT-IR spectral data for terpineol-modified perovskite films in the $4000-500 \mathrm{~cm}^{-1}$ range.

\section{TRPL measurements}

Samples were excited using a Clark-MXR CPA 2001 laser that outputs $780 \mathrm{~nm}$ light with a pulse width of $150 \mathrm{fs}$ at a rate of 1000 pulses/second. This light is passed through a Light Conversion TOPAS to generate the $480 \mathrm{~nm}$ excitation wavelength. TRPL kinetics was obtained using an Optronis streak camera.

\section{Results and discussions}

\section{Moisture stability of terpineol-modified perovskite film}

The moisture stability of the perovskite film at RH $70 \%$ for $3,10,30$, and 70 days was investigated by tracking the phase changes using the XRD analysis. The XRD patterns of the sample at five different times were labeled for a comparison with the calculated XRD pattern using the MDI Jade software, as shown in Fig. 1.

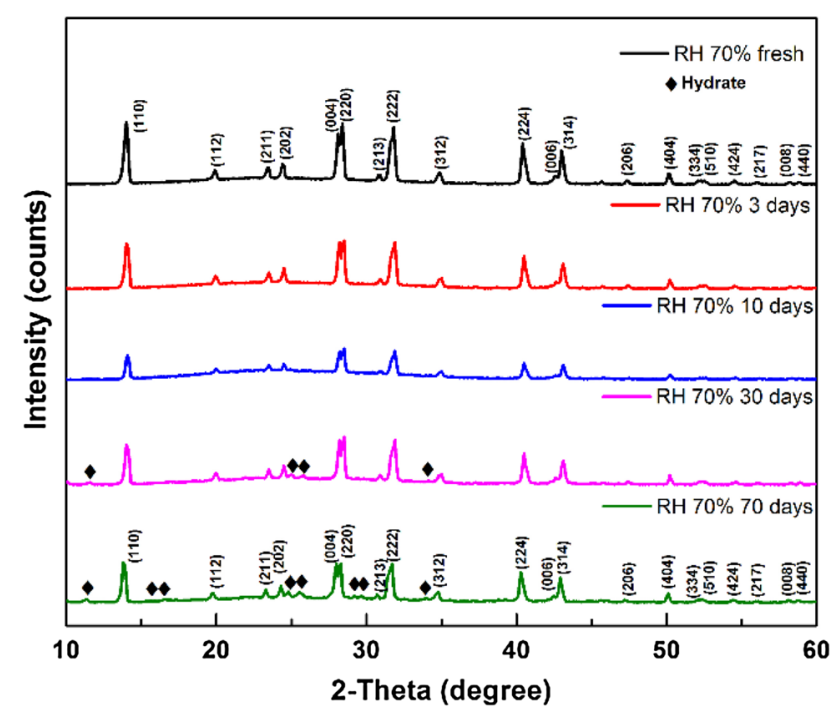

Fig. 1 XRD patterns of fresh perovskite film and stored at RH $70 \%$ for $3,10,30$, and 70 days. The hydrate phase is present for the film at 30 days and increases slowly for 70 days storage 
Showing that typical peaks at $14.10^{\circ}, 23.47^{\circ}, 28.42^{\circ}$, and $30.89^{\circ}$ corresponding to miller planes (110), (211), (220), and (213) of the tetragonal perovskite phase are present in all the cases. The peak at $28.15^{\circ}$ also becomes visible which corresponds to (004). Before 10 days, there are almost no impurity phases detected, indicating the strong moisture resistance of the terpineol-modified perovskite film. There are also unidentified peaks at $11.34^{\circ}$, $16.70^{\circ}, 17.32^{\circ}, 24.74^{\circ}, 25.56^{\circ}$, etc. that showed up in 30 and 70 days. The unidentified peaks did not match either the pure $\mathrm{PbI}_{2}$ or MAI tetragonal phase, which is stable at room temperature. This indicated a possible formation of the hydrate intermediate compound and by comparing with the calculated XRD data of $\left(\mathrm{CH}_{3} \mathrm{NH}_{3}\right)_{4} \mathrm{PbI}_{6} \cdot 2 \mathrm{H}_{2} \mathrm{O}$, those peaks could be identified [33]. This suggests that the water molecules could penetrate into the crystal lattice under high vapor pressure and produce the hydrate compound at $70 \%$ humidity level. A slow growth of the hydrate phase inside the film reflects not only the moisture stability of the terpineol-modified perovskite but also the hydrate phase at the sub-surface can reach equilibrium with both the water vapor at the top surface and the inner perovskite phase resulting in difficulty of the further growth of hydrate compound. For comparison to perovskite films prepared using the more common spin-coating technique, samples were prepared by spin-coating perovskite on to glass and were stored under RH $70 \%$. In stark contrast, the spincoated sample placed under RH $70 \%$ was already degraded into the $\mathrm{PbI}_{2}$ phase and hydrate phase in only 1 day and almost completely degraded in 3 days, as shown in Figure S2.

The second set of experiments was the moisture stability of the perovskite film under RH $50 \%$ for the same time scale as above. The XRD patterns of the sample in five different times are shown in Fig. 2 and were labeled for a comparison with the calculated XRD patterns of perovskite phases.

It can be found that almost no impurity phases existed before 10 days, indicating the strong moisture resistance of the terpineol-modified perovskite film. After that, the lead iodide phase appears only after 30 and 70 days storage at $50 \%$ humidity level. The formation of the $\mathrm{PbI}_{2}$ phase inside the film originates from the diffusion of intercalated polar $\mathrm{CH}_{3} \mathrm{NH}_{3}{ }^{+}$away from the inorganic $\mathrm{PbI}_{2}$ cage with the aid of water molecules. The fact that the $\mathrm{PbI}_{2}$ phase grows slowly inside the film which also provides evidence for the moisture stability of the terpineol-modified perovskite.

The perovskite film exhibited excellent stability when stored at $30 \% \mathrm{RH}$ in the same time scale. The XRD patterns of the sample stored for five different times are shown in Fig. 3, displaying that no detectable impurity phases existed inside the perovskite film after storage for 70 days.

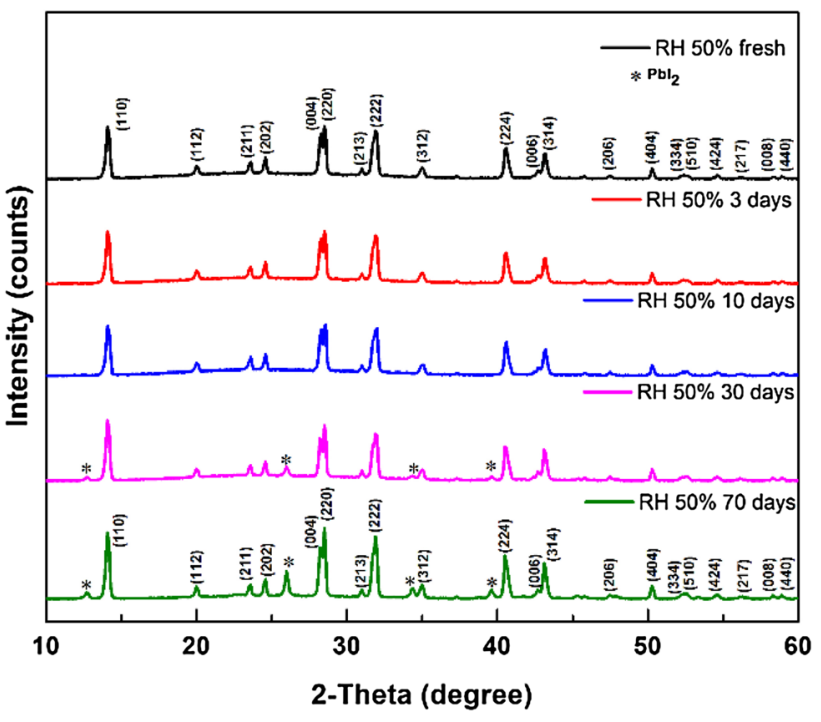

Fig. 2 XRD patterns of fresh perovskite film and stored at RH $50 \%$ for $3,10,30$, and 70 days. The $\mathrm{PbI}_{2}$ phase is present in the film stored for 30 days and increases slowly after 70 days storage

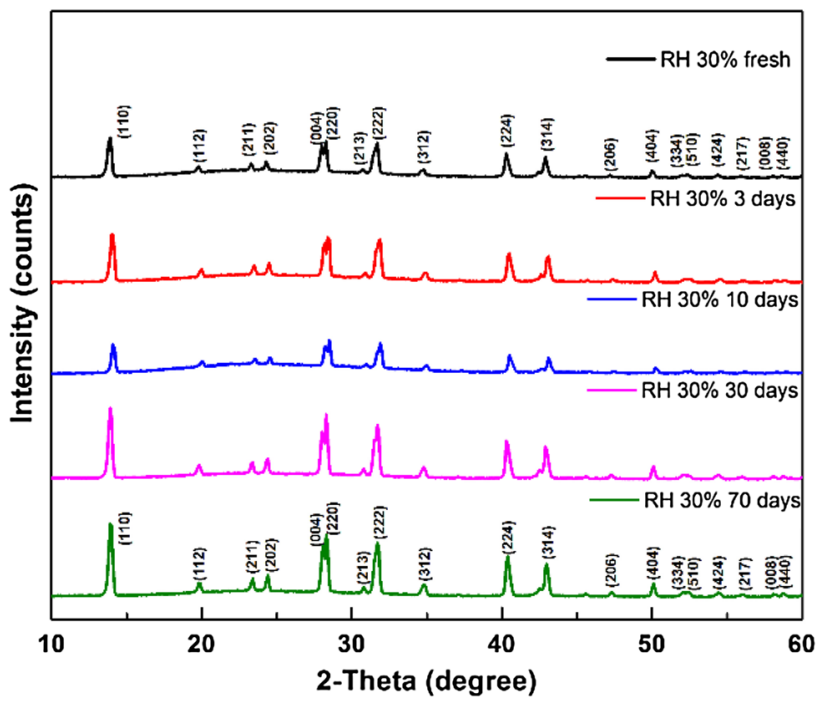

Fig. 3 XRD patterns of fresh perovskite film and stored at RH $30 \%$ for $3,10,30$, and 70 days. Almost no impurity phases are present in the film in all cases

The XRD patterns of samples under RH30, 50, $70 \%$ for 10, 30, and 70 days, respectively, were shown in Figure S3-S5.

The SEM and EDX can also confirm the XRD analysis. The SEM images and EDX spectrum of the fresh sample are shown in Fig. 4. The perovskite grains were observed to be polygons with sharp outlines and facets in different sizes. The EDX spectrum of the overview region shows the composition of perovskite. There are slightly larger deviation errors with the concentration of $\mathrm{C}$ and $\mathrm{N}$ probably due to the light element and small electron scattering cross section. 
The SEM and EDX elemental mapping of perovskite film after 70 days storage at RH70 \% are shown in Fig. 5. The morphology of the fresh perovskite grains changed from sharp outlines to a more rounded shape like melting after long storage time under $70 \%$ moisture atmosphere. The grain size on average also becomes smaller. Through a square scan of the EDX mapping on the bulk segregation, it can be found that the oxygen exists in the film which is further evidence for the presence of the hydrate compound detected in the XRD measurement.

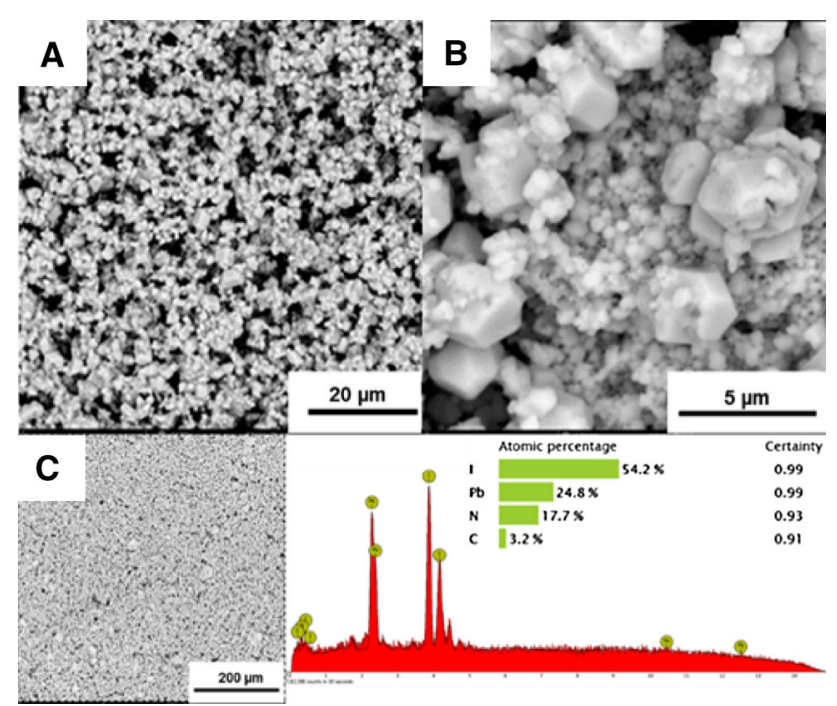

Fig. 4 SEM images and EDX spectrum of the fresh perovskite film, $\mathbf{a}$ at $20 \mu \mathrm{m}$ scale, and $\mathbf{b}$ at $5 \mu \mathrm{m}$ scale, and $\mathbf{c}$ EDX spectrum acquired by overview scanning of image c, scale bar is $200 \mu \mathrm{m}$

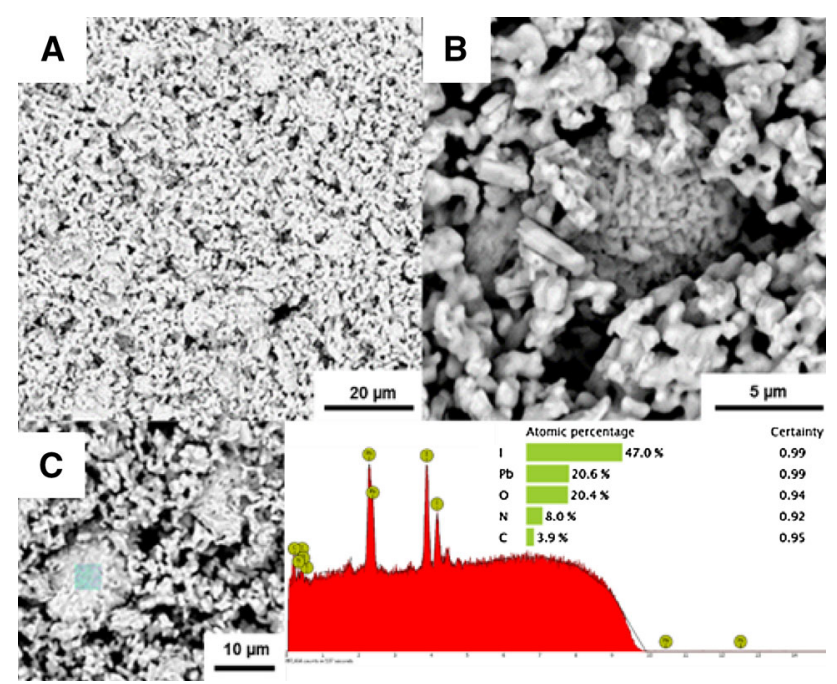

Fig. 5 SEM images and EDX spectrum of the degraded regions in the perovskite film stored at RH $70 \%$ for 70 days, a at $20 \mu \mathrm{m}$ scale, and $\mathbf{b}$ at $5 \mu \mathrm{m}$ scale, and $\mathbf{c}$ EDX spectrum acquired in a square area of image c, scale bar is $10 \mu \mathrm{m}$
Similar observation of the sample stored at RH $50 \%$ for 70 days is shown in Fig. 6. The morphology of the perovskite grains changed from sharp outlines for the fresh state to a less defined shape after long storage time under $50 \%$ moisture atmosphere. Through a rectangle scan of the EDX mapping on the honeycomb bulk body, only elements $\mathrm{Pb}$ and I can be found that well confirm the presence of surface rich $\mathrm{PbI}_{2}$ phase detected in the XRD measurement.

The SEM images and EDX spectrum of the sample stored at RH $30 \%$ for 70 days are shown in Fig. 7. There

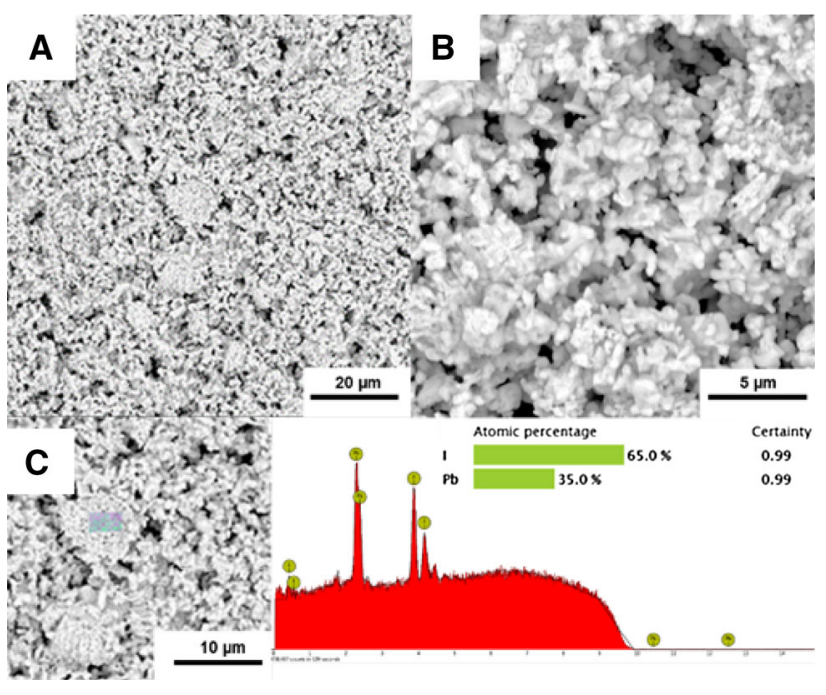

Fig. 6 SEM images and EDX spectrum of the degraded regions in the perovskite film stored at RH $50 \%$ for 70 days, a at $20 \mu \mathrm{m}$ scale, and $\mathbf{b}$ at $5 \mu \mathrm{m}$ scale, and $\mathbf{c}$ EDX spectrum acquired in a square area of image c, scale bar is $10 \mu \mathrm{m}$

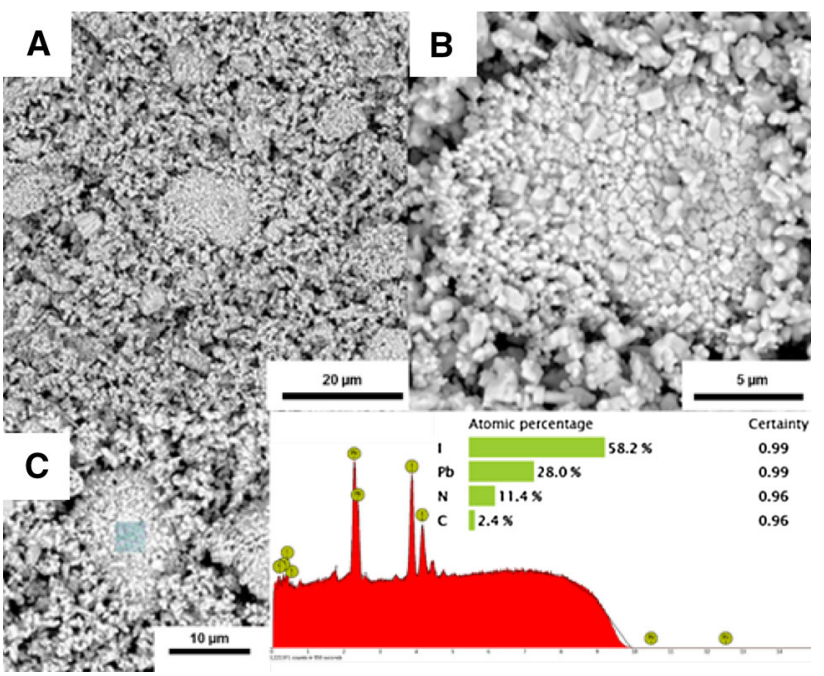

Fig. 7 SEM images and EDX spectrum of the perovskite film stored at RH $30 \%$ for 70 days, a at $20 \mu \mathrm{m}$ scale, and $\mathbf{b}$ at $5 \mu \mathrm{m}$ scale, and c EDX spectrum acquired in a square area of image c, scale bar is $10 \mu \mathrm{m}$

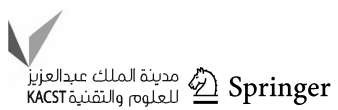


are no major differences in the grain morphology observed between the sample stored at RH $30 \%$ and the fresh one.

The constituents of the paste evolved during the stirring process before the final formation of perovskite phase. From the XRD patterns in Figure S6, the black perovskite paste is not pure and contains the intermediate phase and $\mathrm{PbI}_{2}$ in the first few hours. The intermediate phase might be the coordination compound of terpineol and MAI. With the longer stirring time, the tetragonal perovskite phase coexisted with only $\mathrm{PbI}_{2}$ phase in 1 day and became very pure with almost no $\mathrm{PbI}_{2}$ observed after 3 days of stirring.

The presence of terpineol in the pervoskite film and the interaction between them are further confirmed from the Fourier transform-infrared (FT-IR) spectrum. Figure 8 shows the IR spectra for pure terpineol solvent and the freshly prepared perovskite film made from the perovskite paste. It can be clearly observed that terpineol molecules were contained within the perovskite film, while the typical peaks of $\mathrm{N}-\mathrm{H}$ and $\mathrm{C}-\mathrm{H}$ vibrations for perovskite can also be identified. It is worth noting that the stretching vibration of $\mathrm{O}-\mathrm{H}$ in terpineol appeared at $3374 \mathrm{~cm}^{-1}$ is shifted to lower wavenumber of $3350 \mathrm{~cm}^{-1}$. The shift of the $\mathrm{O}-\mathrm{H}$ peak of terpineol is due to an interaction between the $-\mathrm{OH}$ group and Lewis acids of $\mathrm{MA}^{+}$ions in the perovskite film [27]. In the harmonic approximation using a diatomic model, the frequency of vibration is proportional to the square root of the force constant [28]. Thus, the red shift of
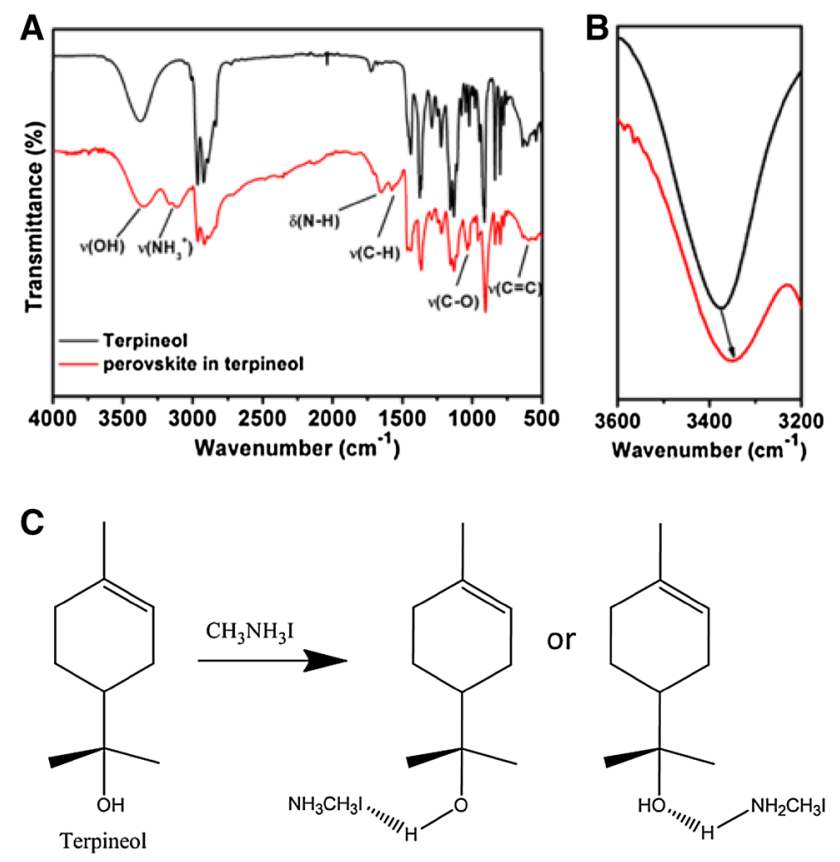

Fig. 8 Fourier transform-infrared spectra (FT-IR) of a pure terpineol and perovskite film made from the perovskite paste, $\mathbf{b}$ expanded fingerprint region for the $\mathrm{O}-\mathrm{H}$ vibrations, and $\mathbf{c}$ schematic of possible interactions between MAI and terpineol along with their chemical structures
$\mathrm{O}-\mathrm{H}$ stretching frequency indicates a lower force constant, which is due to a decreased bond strength between oxygen and hydrogen as a consequence of the adduct formation and interactions with $\mathrm{MA}^{+}$or $\mathrm{I}^{-}$ions. This observation further suggests the effective passivation of terpineol in this system.

\section{Transient PL measurement for carrier lifetime}

The carrier lifetime is an important parameter for developing perovskite-based optoelectronic devices. It has a direct relation to the defect formation and carrier transport properties, and thus reflects the materials quality. To prove that the terpineol-passivated perovskite film has a good stability without a compromise of good carrier lifetimes, time-resolved photoluminescence measurements were employed. Figure 9 shows the kinetic decay curves monitored at $\lambda_{\mathrm{PL}}$ of $780 \mathrm{~nm}$. The PL decay of the fresh perovskite film could be fitted with a single exponential decay equation and a PL lifetime of 16.9 ns was attained. All samples that were exposed to humidity were fit with a multi-exponential decay equation. The fact that two time constants were required to fit the decay curve suggests that there is some decay in these films after being exposed to moisture. However, based on the structural analysis above, this degradation should be minimal. All samples stored under a humid atmosphere, except for two, which show relatively the same time constants. The fast component ranged from 3 to $5 \mathrm{~ns}$ and the slower component ranged from 13 to $18 \mathrm{~ns}$. The longer time constants increased with increased percentages of humidity. The two exceptions to the lifetime trend above are the films stored under 50 and
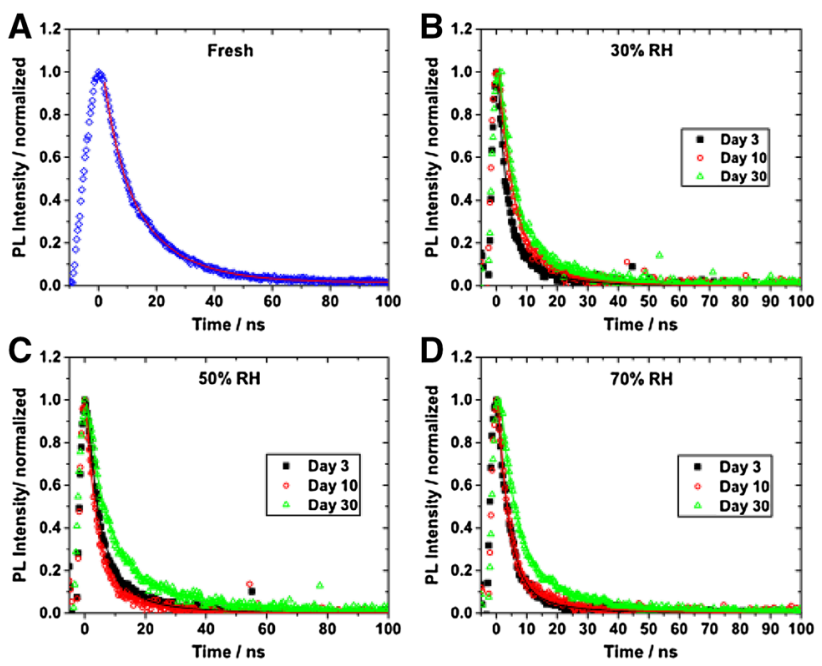

Fig. 9 a TRPL decay dynamics of the freshly prepared perovskite film. b-d TRPL decay dynamics of perovskite films stored under 30, 50 , and $70 \%$ relative humidity, respectively. Films were stored for 3 (black squares), 10 (red circles), and 30 (green triangles) days. PL decay lifetimes are reported in Table 1 
$70 \%$ humidity for 30 days. The sample stored at $50 \%$ humidity for 30 days shows the evidence of degradation in the XRD and this leads to the formation of $\mathrm{PbI}_{2}$ on the surface of the perovskite grains. $\mathrm{PbI}_{2}$ has been shown to act as a passivating layer that can prolong charge carrier recombination [23]. Here, we find that the lifetime is extended from 15 to $36 \mathrm{~ns}$ when $\mathrm{PbI}_{2}$ is present in the film. The sample stored at $70 \% \mathrm{RH}$ for 30 days shows longer charge carrier recombination times, as well. This result in conjunction with the XRD analysis indicates that the hydrate phase also acts as passivating layer which leads to an increase of the PL decay time from 17 ns to 22 ns when the hydrate is present. This effect may also explain why an increase of the PL lifetime is observed when the perovskite films are stored under more humid atmospheres. Typically, the surface modifications of perovskite are performed during the solution preparation or annealing step [29, 30]. However, the findings of this work show that when minimal surface modifications occur due to degradation, surface states can become passivated leading to prolonged charge carrier recombination lifetimes. The corresponding timeresolved photoluminescence contours are shown in Figure $\mathrm{S} 7$ and $\mathrm{S} 8$.

\section{Conclusions}

We demonstrated a novel method to produce perovskite paste by dispersing equimolar mixed $\mathrm{PbI}_{2}$ and MAI powders into the terpineol by way of stirring. The perovskite film prepared by blading the paste showed very good moisture stability. By placing the samples at three different moisture levels, it can be found that all three samples exhibit good stability at long time exposure to moisture for 70 days. The XRD and SEM/EDX results showed that there is a small amount of hydrate compound in the sample stored at $\mathrm{RH} 70 \%$ for 70 days, while the $\mathrm{PbI}_{2}$ as the degraded phase was observed in the sample stored at $\mathrm{RH}$ $50 \%$. However, the sample stored at RH $30 \%$ exhibited almost no change in the meantime. The modified perovskite film by hydrophobic terpineol molecules presented significantly enhanced moisture stability. Water diffusion into perovskite crystal lattice was effectively blocked chemically by the hydrophobic repulsion at the molecular level and physically by the layer encapsulation on a macroscopic scale. Time-resolved photoluminescence spectroscopy revealed that there was some degradation of perovskite films stored under the humid conditions. However, XRD and SEM reveal that this degradation is quite minimal. In the most humid atmospheres, prolonged charge carrier recombination lifetimes due to the passivation of surface states by the formation of either a $\mathrm{PbI}_{2}$ or a hydrated perovskite rich surface are observed.
Acknowledgments The authors acknowledge helpful discussions with Prof. Y. Zhao from Shanghai Jiaotong University and thank WeiChun Lin for the assistance with film fabrications.

Open Access This article is distributed under the terms of the Creative Commons Attribution 4.0 International License (http:// creativecommons.org/licenses/by/4.0/), which permits unrestricted use, distribution, and reproduction in any medium, provided you give appropriate credit to the original author(s) and the source, provide a link to the Creative Commons license, and indicate if changes were made.

\section{References}

1. Chavhan, S., Miguel, O., Grande, H.-J., Gonzalez-Pedro, V., Sánchez, R.S., Barea, E.M., Mora-Seró, I., Tena-Zaera, R.: Organo-metal halide perovskite-based solar cells with $\mathrm{CuSCN}$ as the inorganic hole selective contact. J. Mater. Chem. A 2, 12754-12760 (2014)

2. Zhao, Y., Zhu, K.: Charge transport and recombination in perovskite $\left(\mathrm{CH}_{3} \mathrm{NH}_{3}\right) \mathrm{PbI}_{3}$ sensitized $\mathrm{TiO}_{2}$ solar cells. J. Phys. Chem. Lett. 4, 2880-2884 (2013)

3. Gao, P., Grätzel, M., Nazeeruddin, M.K.: Organohalide lead perovskites for photovoltaic applications. Energy Environ. Sci. 7, 2448 (2014)

4. Jeon, N.J., Noh, J.H., Yang, W.S., Kim, Y.C., Ryu, S., Seo, J., Seok, S.: Compositional engineering of perovskite materials for high-performance solar cells. Nature 517, 476-480 (2015)

5. Zhao, Y., Zhu, K.: Solution chemistry engineering toward highefficiency perovskite solar cells. J. Phys. Chem. Lett. 5, 4175-4186 (2014)

6. Cheng, Z., Lin, J.: Layered organic-inorganic hybrid perovskites: structure, optical properties, film preparation, patterning and templating engineering. Crystal Eng. Commun. 12, 2646-2662 (2010)

7. Heo, J.H., Im, S.H., Noh, J.H., Sarkar, A., Nazeeruddin, M.K., Grätzel, M., Seok, S.: Efficient inorganic-organic hybrid heterojunction solar cells containing perovskite compound and polymeric hole conductors. Nat. Photonics 7, 486-491 (2013)

8. Etgar, L., Gao, P., Xue, Z., Peng, Q., Chandiran, A.L., Liu, B., Nazeeruddin, M.K., Grätzel, M.: Mesoscopic $\mathrm{CH}_{3} \mathrm{NH}_{3} \mathrm{PbI}_{3} /$ $\mathrm{TiO}_{2}$ heterojunction solar cells. J. Am. Chem. Soc. 134(42), 17396-17399 (2012)

9. Zhao, Y., Zhu, K.: Three-step sequential solution deposition of $\mathrm{PbI}_{2}$-free $\mathrm{CH}_{3} \mathrm{NH}_{3} \mathrm{PbI}_{3}$ perovskite. J. Mater. Chem. A 3, 9086 (2015)

10. Jeon, N.J., Noh, J.H., Kim, Y.C., Yang, W.S., Ryu, S.C., Seok, S.: Solvent engineering for high-performance inorganic-organic hybrid perovskite solar cells. Nat. Mater. 13, 897-903 (2014)

11. Ku, Z., Rong, Y., Xu, M., Liu, T., Han, H.: Full printable processed mesoscopic $\mathrm{CH}_{3} \mathrm{NH}_{3} \mathrm{PbI}_{3} / \mathrm{TiO}_{2}$ heterojunction solar cells with carbon counter electrode. Sci. Rep. 3, 3132 (2013)

12. Marchioro, A., Teuscher, J., Friedrich, D., Kunst, M., van de Krol, R., Moehl, T., Grätzel, M., Moser, J.E.: Unravelling the mechanism of photoinduced charge transfer processes in lead iodide perovskite solar cells. Nat. Photonics 8, 250-255 (2014)

13. Zhu, Z., Ma, J., Wang, Z., Mu, C., Fan, Z., Du, L., Bai, Y., Fan, L., Yan, H., Phillips, D.L., Yang, S.: Efficiency enhancement of perovskite solar cells through fast electron extraction: the role of graphene quantum dots. J. Am. Chem. Soc. 136, 3760-3763 (2014)

14. Miyata, A., Mitioglu, A., Plochocka, P., Portugall, O., Wang, J.T., Stranks, S.D., Snait, H.J., Nicholas, R.J.: Direct 
measurement of the exciton binding energy and effective masses for charge carriers in organic-inorganic tri-halide perovskites. Nat. Phys. 11, 582-587 (2015)

15. Zhao, Y., Nardes, A.M., Zhu, K.: Solid-state mesostructured perovskite $\mathrm{CH}_{3} \mathrm{NH}_{3} \mathrm{PbI}_{3}$ solar cells: charge transport, recombination, and diffusion length. J. Phys. Chem. Lett. 5, 490-494 (2014)

16. Qin, P., Tanaka, S., Ito, S., Tetreault, N., Manabe, K., Nishino, H., Nazeeruddin, M.K., Grätzel, M.: Inorganic hole conductorbased lead halide perovskite solar cells with $12.4 \%$ conversion efficiency. Nat. Commun. 5, 3834 (2014)

17. Park, N.-G.: Organometal perovskite light absorbers toward a $20 \%$ efficiency low-cost solid-state mesoscopic solar cell. J. Phys. Chem. Lett. 4, 2423-2429 (2013)

18. Kim, J.H., Williams, S.T., Cho, N., Chueh, C., Jen, A.K.-Y.: Enhanced environmental stability of planar heterojunction perovskite solar cells based on blade-coating. Adv. Energy Mater. 5, 1401229 (2015)

19. Niu, G., Li, W., Meng, F., Wang, L., Dong, H., Qiu, Y.: Study on the stability of $\mathrm{CH}_{3} \mathrm{NH}_{3} \mathrm{PbI}_{3}$ films and the effect of post-modification by aluminum oxide in all-solid-state hybrid solar cells. J. Mater. Chem. A 2, 705 (2014)

20. Leguy, A.M.A., Hu, Y., Campoy-Quiles, M., Alonso, M.I., Weber, O.J., Azarhoosh, P., van Schilfgaarde, M., Weller, M.T., Bein, T., Nelson, J., Docampo, P., Barnes, P.R.F.: Reversible hydration of $\mathrm{CH}_{3} \mathrm{NH}_{3} \mathrm{PbI}_{3}$ in films, single crystals, and solar cells. Chem. Mater. 27, 3397-3407 (2015)

21. Niu, G., Guo, X., Wang, L.: Review of recent progress in chemical stability of perovskite solar cells. J. Mater. Chem. A 3, 8970-8980 (2015)

22. Li, W., Li, J., Wang, L., Niu, G., Gao, R., Qiu, Y.: Post modification of perovskite sensitized solar cells by aluminum oxide for enhanced performance. J. Mater. Chem. A 1, 11735-11740 (2013)
23. Wang, L., McCleese, C., Kovalsky, A., Zhao, Y., Burda, C.: Femtosecond time-resolved transient absorption spectroscopy of $\mathrm{CH}_{3} \mathrm{NH}_{3} \mathrm{PbI}_{3}$ perovskite films: evidence for passivation effect of $\mathrm{PbI}_{2}$. J. Am. Chem. Soc. 136(35), 12205-12208 (2014)

24. Noel, N.K., Abate, A., Stranks, S.D., Parrott, E., Burlakov, V., Goriely, A., Snaith, H.J.: Enhanced photoluminescence and solar cell performance via lewis base passivation of organic-inorganic lead halide perovskites. ACS Nano 8(10), 9815-9821 (2014)

25. Smith, I.C., Hoke, E.T., Solis-Ibarra, D., McGehee, M.D., Karunadasa, H.I.: A layered hybrid perovskite solar-cell absorber with enhanced moisture stability. Angew. Chem. Int. Ed. 53, 1-5 (2014)

26. Hwang, I., Jeong, I., Lee, J., Ko, M.J., Yong, K.: Enhancing stability of perovskite solar cells to moisture by the facile hydrophobic passivation. Appl. Mater. Interfaces 7(31), 17330-17336 (2015)

27. Ahn, N., Son, D.Y., Jang, I.H., Kang, S.M., Choi, M., Park, N.G.: Highly reproducible perovskite solar cells with average efficiency of $18.3 \%$ and best efficiency of $19.7 \%$ fabricated via lewis base adduct of lead(II) iodide. J. Am. Chem. Soc. 137, 8696-8699 (2015)

28. Colthup, N.B., Daly, L.H., Wiberley, S.E.: Introduction to Infrared and Raman Spectroscopy, 2nd edn. Elsevier, New York (2012)

29. Chen, Q., Zhou, H., Song, T.-B., Luo, S., Hong, Z., Duan, H.-S., Dou, L., Liu, Y., Yang, Y.: Controllable self-induced passivation of hybrid lead iodide perovskites toward high performance solar cells. Nano Lett. 14, 4158-4163 (2014)

30. Eperon, G.E., Habisreutinger, S.N., Leijtens, T., Bruijnaers, B.J., Van Franeker, J.J., deQuilettes, D.W., Pathak, S., Sutton, R.J., Grancini, G., Ginger, D.S., Janssen, R.A.J., Petrozza, A., Snaith, H.J.: The importance of moisture in hybrid lead halide perovskite thin film fabrication. ACS Nano 9, 9380-9393 (2015) 